\title{
Retraction Note: Determinants of suboptimal breastfeeding practice in Debre Berhan town, Ethiopia: a cross sectional study
}

Teklemariam Gultie ${ }^{1 *}$ and Girum Sebsibie ${ }^{2}$

\section{Retraction note}

The authors are retracting this article [1] because there is significant overlap of both text and data with the Master's Thesis of Hilina Ketma, "Assessment of prevalence and determinants of suboptimal breast feeding among mothers of children aged less than two years in Dire Dawa City Administration, Ethiopia, June 2013", which was defended at the School of Graduate Studies, Addis Ababa University, in June 2013.

\footnotetext{
Author details

'Department of Midwifery, College of Medicine and Health Science, Arba Minch University, Arba Minch, Ethiopia. ${ }^{2}$ Department of Nursing and

Midwifery, College of Health Science, Addis Ababa University, Addis Ababa,

Ethiopia.
}

Received: 6 February 2018 Accepted: 23 February 2018

Published online: 07 March 2018

\section{Reference}

1. Gultie T, Sebsibie G. Determinants of suboptimal breastfeeding practice in Debre Berhan town, Ethiopia: a cross sectional study. Int Breastfeed J. 2016; 11:5. https://doi.org/10.1186/s13006-016-0063-z.

\footnotetext{
* Correspondence: tekledb2002@gmail.com

'Department of Midwifery, College of Medicine and Health Science, Arba

Minch University, Arba Minch, Ethiopia
} 